BNL - 68561

Informal Report

CAP-314-RF-R

\title{
A Study of Rapid Cavity Tuning
}

Yongxiang Zhao

Brookhaven National Laboratory

July 2001 


\title{
A STUDY OF RAPID CAVITY TUNING
}

\author{
Yongxiang Zhao \\ Physics Department, Bldg. 901-A \\ Brookhaven National Laboratory, Upton, NY 11973
}

\begin{abstract}
An FFAG moot likely requires rapid cavity tuning. The cavity must also have a very high gradient. To satisfy both the high power and rapid tuning requirements is a big challenge. Detailed investigation of the possibility is addressed. Included are general thoughts, dual-loop and simple loop analyses, and a study of using ferrite or PIN diodes. Also proposed is a phase control scheme, which may be a better solution if the needed components can be developed. Finally, an energy analysis reveals the difficult: of high power tuning.
\end{abstract}

\section{THE ISSUE}

The muon collider requires rapid acceleration. The accelerating gradient must be as high as possible. In order to save power, superconducting cavities are preferable. One of the acceleration options is an FFAG (fixed field alternating gradient). Acceleration takes place over many turns around the ring without any change in magnetic fields. However, since the momentum changes every turn, the rotation time changes too. The change depends on the lattice design. In order for the rf accelerating wave to be synchronized with the beam, one must change the $\mathrm{RF}$ phase or frequency correspondingly.

Assume that the relationship between revolution time and the momentum is shown qualitatively in Fig.1, and the range of the momentum is set in a linear area such that:

$$
\frac{d \tau}{d E}=s=\text { const }
$$

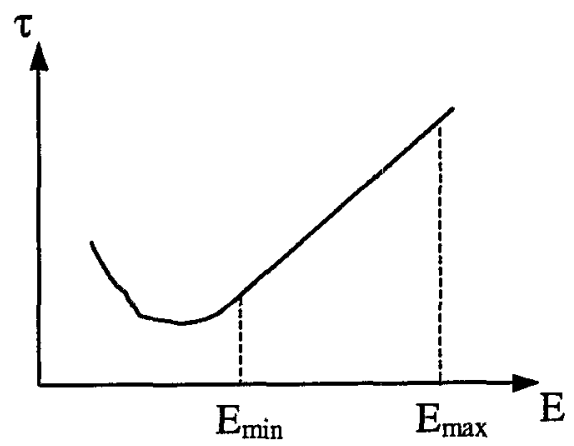

Fig.1 Revolution time vs momentum

Further assume that the beam energy increases equally in every turn by $\Delta E$. Then in $n^{\text {th }}$ turn the increment of rotation time is:

$$
\Delta \tau_{n}=s n \Delta E=n \Delta \tau_{1}
$$

If the frequency remains constant, then the phase should be shifted by:

$$
\omega \Delta \tau_{n}=\omega n \Delta \tau_{l}
$$

But the total phase shifit should be:

$$
\Delta \Phi_{n}=\omega\left(\Delta \tau_{1}+\Delta \tau_{2}+\ldots \ldots \ldots+\Delta \tau_{n}\right)=\omega \Delta \tau_{1}(1+2+\ldots \ldots .+n)=\omega \Delta \tau_{1} \frac{\left(n^{2}+n\right)}{2}
$$

It turns out that the phase is changed with turn's number squared. On the other hand, if one changes the frequency to keep the circumference harmonic number $h$ constant 


$$
\omega \tau_{n}=2 \pi h=\text { constant }
$$

then $\frac{\Delta f_{n}}{f}=-\frac{\Delta \tau_{n}}{\tau}$,

meaning that the frequency will change linearly with time.

In principle changing frequency and changing phase can be equivalent, but the engineering set up may be quite different. As a numerical example, a design gives roughly the following data ${ }^{[1]}$ :

$\begin{array}{ll}\text { Frequency } & 200 \mathrm{MHz} \\ \text { Revolution time } & 768 \mathrm{~ns} \text { (at starting energy) } \\ \Delta \tau / \text { per turn } & \sim 0.2 \mathrm{~ns} / \text { turn }\end{array}$

Assuming the FFAG scheme involves a total of 10 cycles, the following table lists the relevant parameters.

Table 1

\begin{tabular}{|c|c|c|c|c|c|c|}
\hline $\begin{array}{l}\text { Turn's } \\
\text { number }\end{array}$ & \multicolumn{2}{|c|}{$\begin{array}{c}\text { Time shift } \\
\text { (ns) }\end{array}$} & \multicolumn{2}{|c|}{$\begin{array}{c}\text { Phase shift } \\
\text { (Fixed frequency) }\end{array}$} & \multicolumn{2}{|c|}{ Frequency shift } \\
\hline $\mathrm{n}$ & $\Delta \tau_{\mathrm{r}}$ & $\Sigma \Delta \tau_{\mathrm{n}}$ & $\Sigma \Delta \phi_{\mathrm{n}} / 2 \pi$ & $\Sigma \Delta \phi_{n}(\operatorname{deg})$ & $\Delta \mathrm{f}_{\mathrm{n}} / \mathrm{f}$ & $\Delta \mathrm{f}_{\mathrm{n}}(\mathrm{MHz})$ \\
\hline 0 & 0 & 0 & 0 & 0 & 0 & 0 \\
\hline 1 & 0.2 & 0.2 & 0.04 & 14.4 & $-2.60 \times 10^{-4}$ & -0.052 \\
\hline 2 & 0.4 & 0.6 & 0.12 & 43.2 & $-5.21 \times 10^{-4}$ & -0.104 \\
\hline 3 & 0.6 & 1.2 & 0.24 & 86.4 & $-7.81 \times 10^{-4}$ & -0.156 \\
\hline 4 & 0.8 & 2.0 & 0.40 & 144.0 & $-1.04 \times 10^{-3}$ & -0.208 \\
\hline 5 & 1.0 & 3.0 & 0.60 & 216.0 & $-1.30 \times 10^{-3}$ & -0.260 \\
\hline 6 & 1.2 & 4.2 & 0.84 & 302.4 & $-1.56 \times 10^{-3}$ & -0.313 \\
\hline 7 & 1.4 & 5.6 & 1.12 & 403.2 & $-1.82 \times 10^{-3}$ & -0.365 \\
\hline 8 & 1.6 & 7.2 & 1.44 & 518.4 & $-2.08 \times 10^{-3}$ & -0.417 \\
\hline 9 & 1.8 & 9.0 & 1.80 & 648.0 & $-2.34 \times 10^{-3}$ & -0.469 \\
\hline
\end{tabular}

One can see that the total phase shift should be $648^{\circ}$ or roughly 2 cycles if the frequency is kept constant. Or, if changing frequency, it requires about $0.2 \%$ in total. How to realize this tuning is a challenge and will be addressed hereafter.

\section{GENERAL THOUGHTS}

The tuning is an old problem, but the challenge here is two-fold. (1) It must be rapid enough that the tuning can be carried out before the beam returns for the next turn. In the above example, one cycle is less than 1 microsecond. (2) The cavity is operated at a very high voltage, say over one mega-volt. Either of the above requirements can be solved separately, but it is very difficult to meet both requirements simultaneously.

Mechanical tuning probably has the largest capability to handle high power, but it is excluded from consideration due to the rapidity requirement. Even the application of a diaphragm is far slower than needed on a microsecond time scale. 
To tune the driver amplifier chain may be a good option because the bandwidth requirement is modest and the tuning can be accomplished at low level. Either phase or frequency can be easilly changed. But, the load should have a lower $Q$ value to accommodate the rapid change. If the accelerator is a traveling wave structure with its filling time short enough, a tunable amplifier most likely can work. However, the FFAG ring in consideration is a series of cavities forming a linac, so the $Q$ must be very high. Even if the drive frequency of the amplifier can change rapidly, the energy inside the cavity can not be replaced in time.

Since a cavity can not change its frequency rapidly enough by means of changing the drive frequency, one must change the cavity eigenfrequency itself so that the originally stored energy will carry out a free oscillation, of which the frequency varies correspondingly.

Electrical tuning seems a must. Ferrites are commonly used for cavity electric tuning. However, ferrite is usually very lossy, and its capability to withstand high power is doubtful. Besides whether it can be applied at frequencies as high as $200 \mathrm{MHz}$ is also questionable. More detailed properties of ferrite will be addressed later.

Since putting a ferrite inside a common cavity is unlikely to be a solution, in order to minimize the loss, we consider a dual-loop circuit, such that the ferrite is located in the 2nd loop, which has weak coupling with the primary loop. During the charging period, the 2nd loop is far from resonance and little energy goes into it, making the loss tolerable. The $Q$ of the resonator remains high and majority of energy goes into the primary loop. When the beam is coming, the ferrite in the 2 nd loop is properly biased in such a way that the loop becomes near resonance. Consequently, the coupling becomes strong and the eigenfrequency shifts. Although the $Q$ value is then reduced, it doesn't matter as long as the beam passes through without significant field reduction. We'll analyze the dual-loop case next.

A dual-loop circuit usually consists of 2 eigenfrequencies, ignoring high order modes for the moment. Since the case in question is a free oscillation without energy fed in, a transient process analysis is necessary for close viewing of the instantaneous field. Usually there are two frequencies, but the beam senses the composite field. A simulation applying PSpice was done for quantitative analysis.

An alternative is to use PIN diodes. The PIN diode is known to be a good microwave switch. Using PIN diodes to shorten out part of the inductance in a resonant circuit will change its frequency. We'll study its feasibility later.

\section{SUPERFISH SIMULATION OF A CAVITY WITH A.SUBSIDIARY CELL}

If a piece of ferrite is put directly into an accelerator cavity, the $Q$ value will be reduced too much and the loss, as well as the field in the ferrite, will be too high to withstand. So, we consider a 2 nd cell coupled weakly to the primary one, so that it extracts only a small part of the total energy. The requirement of weakness is in consideration of the fact that the primary cavity is superconducting. So we want the energy going to the 2 nd loop to be as low as possible before the ferrite is biased to shift the frequency. 
Fig. 2 shows a scheme. On the bottom is the symmetry axis. On the left is the primary cavity with outer radius of $60 \mathrm{~cm}$, and on the right is the 2 nd cell with weak electrical coupling to the primary. Located at the 2 nd cell is the ferrite, of which the bias is variable to tune its frequency. The variation of the ferrite's permeability will change the electrical length of the cell. For simplicity, the simulation assumes no ferrite but changing the radius of 2 nd cell.

Fig. 3 shows the shape of the model, where $\mathrm{R}_{2}$ is changeable (in Fig. 3 $\mathrm{R}_{2}=50 \mathrm{~cm}$ ). It shows that the radius of the 2nd cell is somewhat less than the primary one, so that it is off the resonance, and the field is weak. From Fig. 4(a), one can see the frequency is almost unchanged due to the existence of the 2 nd cell until near resonance.

Fig.4 summarizes the results of simulations via SUPERFISH. As was expected, the frequency has a perceptible change only when the 2 nd

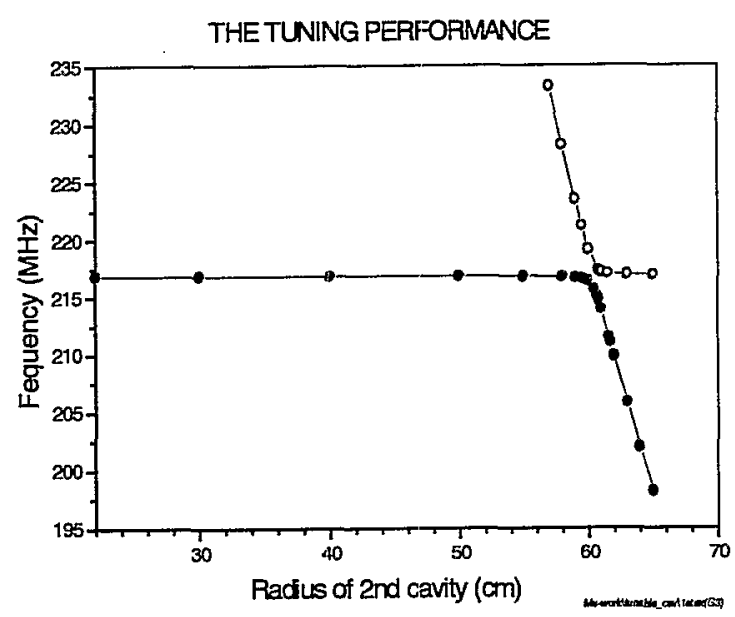

(a)

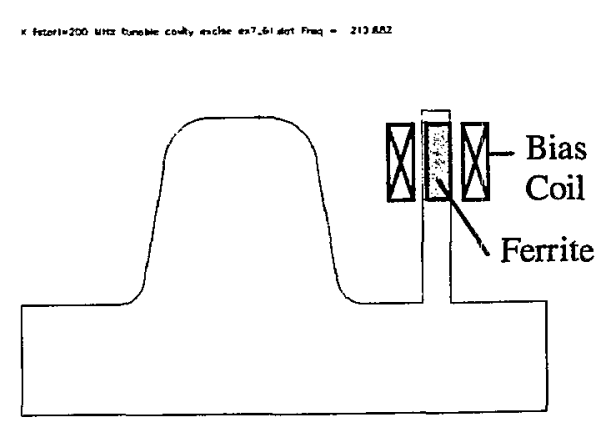

Fig. 2 A cell coupled weakly to the cavity

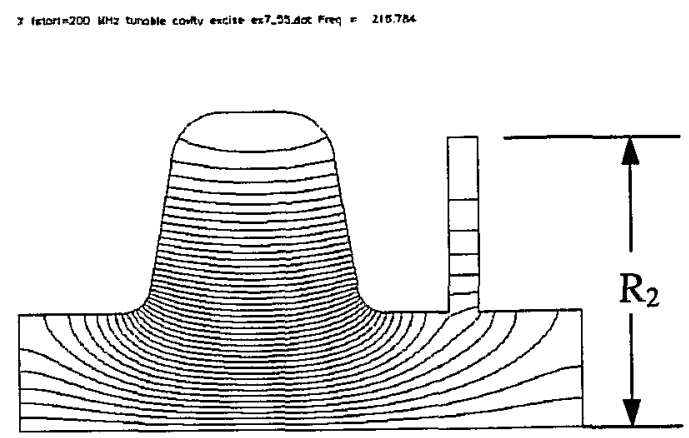

Fig. 3 The model for simulation

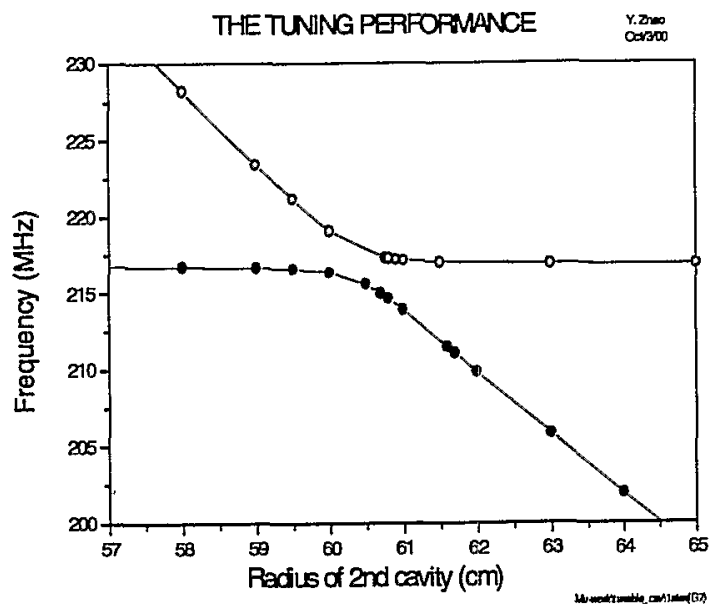

(b)

Fig. 4 The resonant frequencies of a dual-loop circuit (a) and close view (b).

cell is tuned close to the primary frequency where strong coupling occurs. Meanwhile it splits into 2 modes ( 2 eigenfrequencies). Fig.4 (b) is a close view. 
The two modes have different field patterns. According to whether the fields in the two cells are in-phase or opposite phase, they are referred to as 0 -mode and $\pi$-mode respectively. Fig.5 shows two typical ones.

From above results one can see that a tuning range of $1 \%$ is possible. However, difficulties remain. (1) The tuning near resonance is very sensitive, making it hard to control. (2) The field strength and the energy allocation depend on how close the eigenfrequency is to the original drive frequency. If one wants the frequency shifts so much as to close to the 2nd cell's eigenfrequency, the energy will also incline to the 2nd cell. It can also be seen in comparison Fig.5 (a) with Fig.5 (b). The former has more frequency shift than the latter, but the field in the primary cavity is weakened. (3) Two modes exist and only one is desirable. How to avoid the other one is a question. In fact, as described in next section, one can see that it is hard to separate them in a free oscillation. (4) If the primary cavity is superconducting, the magnetic field necessary for biasing the ferrite needs to be cautiously screened.

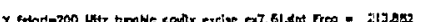

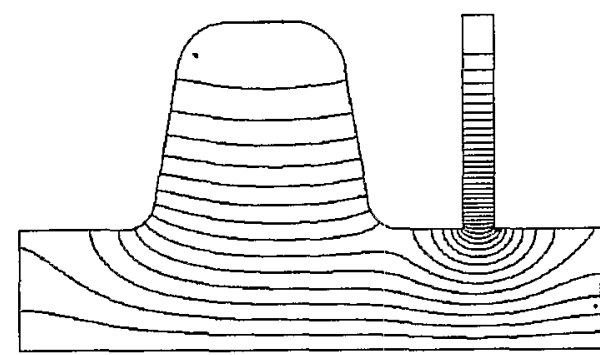

(a) 0-mode $(214 \mathrm{MHz})$

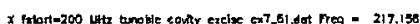

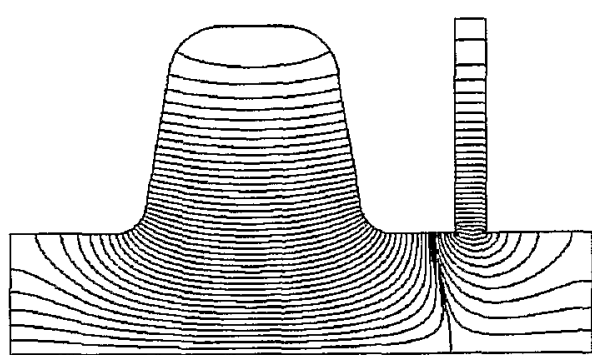

(b ) $\pi$-mode $(217 \mathrm{MHz})$

Fig. 5 The field patterns of different mode

It should be noted that if a normal conducting cavity and rather stronger coupling are employed, 2nd loop with non-resonant status is also applicable, providing the tuning range is narrow enough.

\section{PSPice SIMULATION OF COUPLING LOOP DECAY PROCESS}

The question is arisen due to the fact that the energy being charged inside the cavity will oscillate freely and be independent with the drive frequency when the cavity suddenly change its geometry and its eigenfrequency. We assume the driver is turned off, because it contributes little for free oscillation in a high $Q$ system. In principle, a free oscillation will involve all possible modes, including high order modes. Their distribution depends on the initial and boundary conditions as well as their decay factors.

Let's ignore the high order mode, of which the effect is subsidiary. To apply a simulation we use a coupled two-loop circuit to represent the two-cell cavity with the 2nd loop assumed to contain a lossy ferrite. The eigenfrequency will split into two. One closes to the primary loop and another 2 nd loop. 
The whole process should be like this. At first, the 2nd cavity is detuned too far to influence the primary cavity. The primary cavity is charged and resonant with frequency $f_{0}$ and reaches its saturation. At $t=0$, the ferrite is suddenly biased in such a way that the 2nd cell has its frequency near the primary one. The two cells are then coupled strongly and $f_{0}$ splits into two frequencies $f_{1}$ and $f_{2}$. We assume the driver turns off in the meantime. Now the system will freely oscillate in its own way with two frequencies existing simultaneously. Since $f_{2}$, being more related to 2 nd cell containing ferrite, decays faster than $f_{l}$, eventually the final status is dominated by $f_{l}$, which is slightly different with $f_{0}$.

Both the starting and final states are of single frequency. The interim or transition period is considered a transient process. Unfortunately, this process is so long that the circulated beam must fall in this period. At the beginning, both components are comparable and form a beat wave. We have to study this process carefully.

Obviously that is a problem in the time domain, for which SUPERFISH is not good. In order to do a qualitative analysis, we apply PSpice instead.

(a)

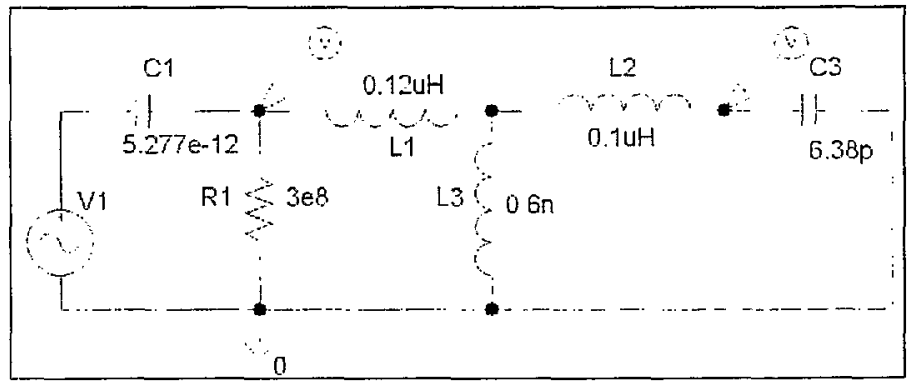

(b)

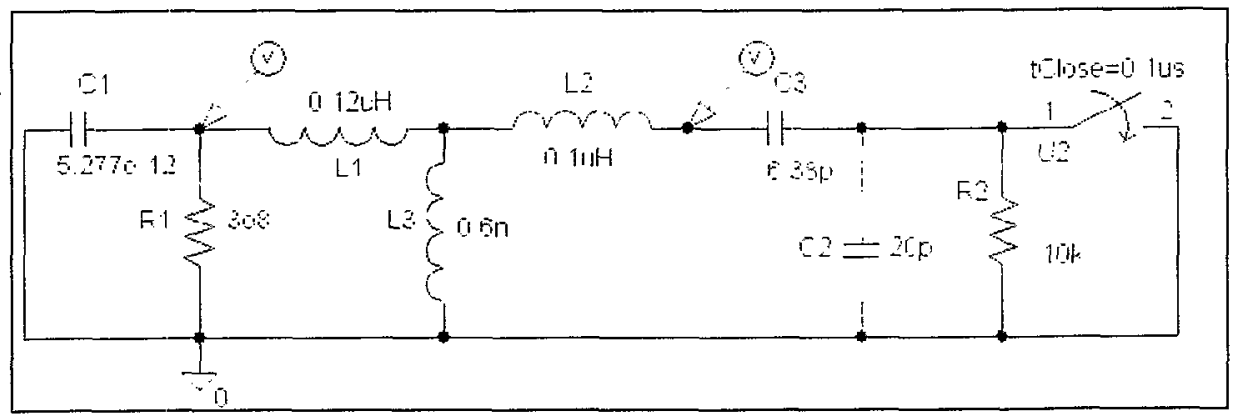

Fig. 6 The circuit to be simulated

Fig.6 shows a 2-loop circuit, where (a) is used to find the eigenfrequencies, i.e. for frequency domain analysis, and (b) for time domain analysis, i.e. simulating the transient process. Both have same parameters when the switch of (b) is closed. The 1st loop consists of $C_{1}, L_{1}$ and $L_{3}$. The 2 nd loop consists of $C_{3}, L_{2}$ and $L_{3}$, where $L_{3}$ is the coupling inductance, corresponding to a coupling coefficient of $k^{2}=L_{3}{ }^{2} / L_{1} L_{2},(k=0.0055)$. For (b), the initial condition is $V=1.0$ at $C_{l}$, with all the rest zero. The switch on the right side is closed at $100 \mathrm{~ns}$. Before $100 \mathrm{~ns}$ the $2 \mathrm{nd}$ loop has a $C_{2}$ in series, which makes the frequency far from the primary one.

Fig. 7 gives a typical simulation result of the transient analysis with a step ceiling time of $0.01 \mathrm{~ns}$. Considering the oscillating period is about $5 \mathrm{~ns}$ (for $f=200 \mathrm{MHz}$ ), this 
means every cycle involves 500 more steps. Coarse steps will cause too much error. Because for time domain the truncation error is cumulative, it is very difficult to get a precise result, especially if too many cycles are involved. The result can only be regarded as a qualitative estimation.

Fig.7 shows the voltages $V_{I}$ at $C_{I}$ (the large green curve) and $V_{2}$ at $C_{3}$ (the smaller red curve). One can see that before $100 \mathrm{~ns}$ (switch not closed) the decay of $V_{1}$ is slow. The voltage $V_{2}$ also exists, though it is much smaller, implying that only a little energy goes to the 2 nd loop. Once the 2 nd loop nears resonance ( $t>100 \mathrm{~ns}$, switch closed ), the energy goes from the 1st loop to the 2 nd loop, back and forth like beat wave. It'll be more obvious if the simulation time lasted longer.

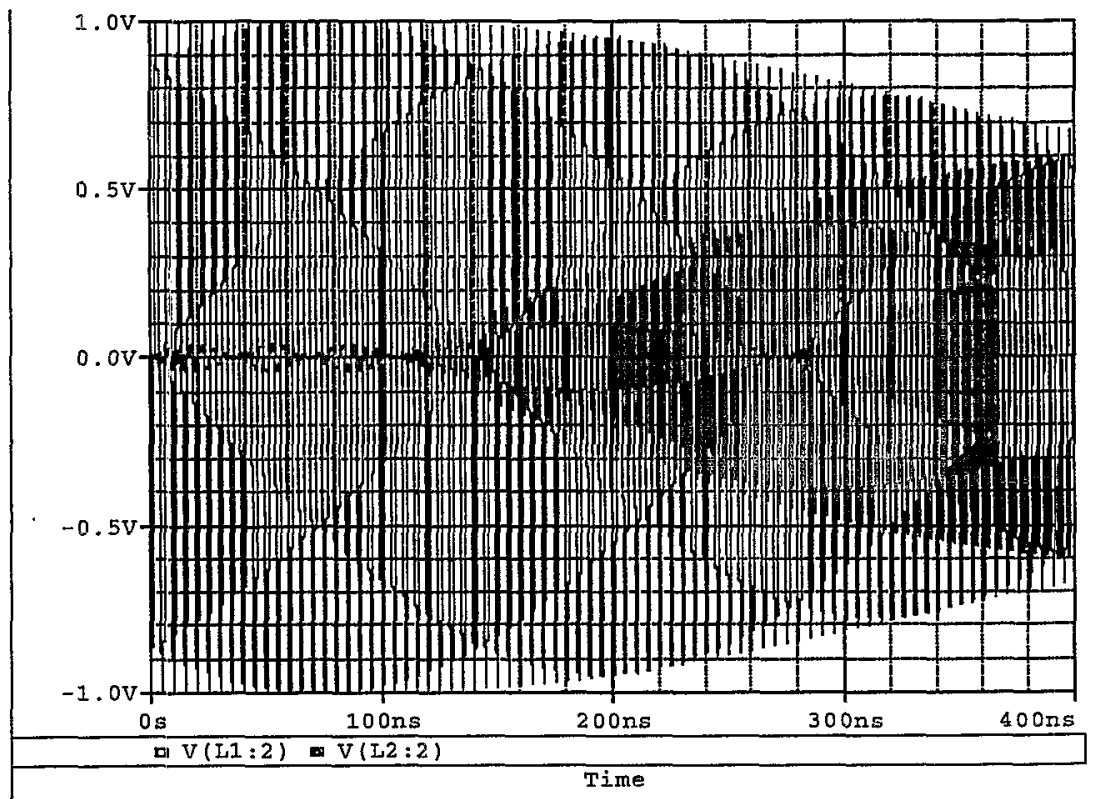

Fig. 7 The oscillating waveform of above circuit

From a close view of Fig.7 we found that the frequency doesn't change much, although there are two frequencies in the frequency domain with a sizable difference.

Table 2 summarizes the data collected from the PSpice runs for the circuit of Fig.6. The first row lists 3 sets of $C_{3}$ values, which change the frequency of the 2nd loop. The 25 rows list two eigenfrequencies resulting from the frequency domain (by circuit Fig. 6(a)) and their difference. The last 4 rows list the frequencies before and after the switch is closed resulting from the time domain (Fig.7) and their difference. The frequencies in the time domain are counted by this way: check the time of two peaks, in between there are $n$ wavelength, then the time difference divided by $n$ is an average period. Its reciprocal is the frequency.

One can see that the two eigenfrequencies, $f_{1}$ and $f_{2}$, differ from each other is in the order of one percent, but in a transient process, the frequency swings in a range of only $6 \times 10^{-4}$ or so. 
Table 2

\begin{tabular}{|c|c|c|c|c|}
\hline \multicolumn{2}{|c|}{ Value $\mathrm{C}_{3}$ in Fig.6 } & $6.38 \mathrm{pF}$ & $6.50 \mathrm{pF}$ & $6.64 \mathrm{pF}$ \\
\hline \multirow{2}{*}{$\begin{array}{c}\text { Frequency (MHz) } \\
\text { (at freq. domain) }\end{array}$} & $\mathrm{f}_{1}$ & 199.774 & 199.609 & 199.569 \\
\cline { 2 - 5 } & $\mathrm{f}_{2}$ & 198.398 & 196.717 & 194.676 \\
\hline Freq. difference & $\Delta \mathrm{f}=\mathrm{f}_{1}-\mathrm{f}_{2}$ & 1.376 & 2.892 & 4.893 \\
\hline Relative span & $\Delta \mathrm{f} / \mathrm{f}_{1}$ & $0.69 \times 10^{-2}$ & $1.45 \times 10^{-2}$ & $2.45 \times 10^{-2}$ \\
\hline Frequency (MHz) & $\mathrm{t}<100 \mathrm{~ns}$ & 199.491 & 199.491 & 199.491 \\
\cline { 2 - 5 } (at time domain) & $\mathrm{t}>10 \dot{\mathrm{ns}}$ & 199.572 & 199.613 & 199.586 \\
\hline Freq. shift & $\Delta \mathrm{f}$ & -0.081 & -0.122 & -0.095 \\
\hline Relative shift & $\Delta \mathrm{f} / \mathrm{f}$ & $4 \times 10^{-4}$ & $6.1 \times 10^{-4}$ & $4.8 \times 10^{-4}$ \\
\hline
\end{tabular}

The most important fact is that what the beam sees is the composite field in some particular instance, that is also what we are interested in. This means that in the above Table the result of time domain is more essential. The frequency of the composite has much less deviation. Surely this is not desirable. Fig. 7 also shows that the energy is swinging back and forth between two loops rather than swinging between two frequencies. The energy swing represents an amplitude modulation like a beat wave. As is well known, a sine wave with amplitude modulation can always be regarded as two side bands in a frequency spectrum analysis. But, the frequency spectrum is not essential in the case under consideration.

It can also be seen that the frequency shift does not change monotonically with the frequency of the 2nd loop (see the last row in Table 2), but existing an optimum point. However, as the data shown are only average, which depends on the chosen time interval, so it can only be regarded as a qualitative reference. We have to analysis it more carefully.

\section{ANALYTIC SOLUTION OF COUPLING LOOP DECAY PROCESS}

As demonstrated in the above section, the frequency split does not mean the frequency will shift from one to another. Nevertheless, the frequency does vary from time to time. The frequency shift shown in the Table 2 is an average during a certain period. Besides, due to the accumulated error, the data shown are not precise. So, a more precise analytic solution is needed.

Fig.8 shows a dual-loop circuit model. Assume that only $C_{1}$ has energy stored at initial condition. Thus:

$$
\begin{aligned}
& t=0, \quad u_{I}=u_{I}(0), u_{2}=0, \\
& u_{1}^{\prime}(0)=\frac{d u_{1}}{d t}=0 ; \quad \frac{d u_{2}}{d t}=0 .
\end{aligned}
$$

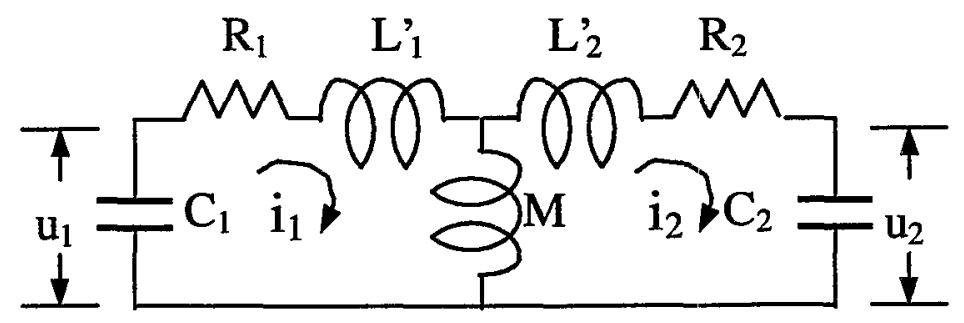

Also assuming:

Fig.8 Dual-loop model for analysis 
$L_{1}=L_{1}^{\prime}+M, \quad L_{2}=L_{2}^{\prime}+M, \quad$ then we can easily get the following equations:

$$
\left\{\begin{array}{l}
L_{1} C_{1} \frac{d^{2} u_{1}}{d t^{2}}+R_{1} C_{1} \frac{d u_{1}}{d t}+u_{1}=M C_{2} \frac{d^{2} u_{2}}{d t^{2}} \\
L_{2} C_{2} \frac{d^{2} u_{2}}{d t^{2}}+R_{2} C_{2} \frac{d u_{2}}{d t}+u_{2}=M C_{1} \frac{d^{2} u_{1}}{d t^{2}}
\end{array}\right.
$$

One can convert it to a more common oscillation equation form:

$$
\left\{\begin{array}{c}
\frac{d^{2} u_{1}}{d t^{2}}+2 \delta_{1} \frac{d u_{1}}{d t}+\omega_{1}^{2} u_{1}=m_{1} \frac{d^{2} u_{2}}{d t^{2}} \\
\frac{d^{2} u_{2}}{d t^{2}}+2 \delta_{2} \frac{d u_{2}}{d t}+\omega_{2}{ }^{2} u_{2}=m_{2} \frac{d^{2} u_{1}}{d t^{2}}
\end{array}\right.
$$

where

$$
\begin{aligned}
& \omega_{1}^{2}=\frac{1}{L_{1} C_{1}}, \omega_{2}^{2}=\frac{1}{L_{2} C_{2}}, \delta_{1}=\frac{R_{1}}{2 L_{1}}=\frac{\omega_{1}}{2 Q_{1}}, \delta_{2}=\frac{R_{2}}{2 L_{2}}=\frac{\omega_{2}}{2 Q_{2}} \\
& m_{1}=\frac{M C_{2}}{L_{1} C_{1}}, m_{2}=\frac{M C_{1}}{L_{2} C_{2}}, k^{2}=m_{1} m_{2}=\frac{M^{2}}{L_{1} L_{2}}
\end{aligned}
$$

For normalization all parameters are dimensionless, substituting:

$$
\tau=\omega_{1} t, \quad d_{1}=\frac{\delta_{1}}{\omega_{1}}=\frac{1}{2 Q_{1}}, d_{2}=\frac{\delta_{2}}{\omega_{1}}=\frac{1}{2 Q_{2}} \cdot \frac{\omega_{2}}{\omega_{1}}, f_{1}=\frac{\omega_{1}}{\omega_{1}}=1, f_{2}=\frac{\omega_{2}}{\omega_{1}}
$$

Note here $f$ are normalized frequencies with $f_{l}=1$, which we retain in the formulas for symmetrization. Then we obtain:

$$
\left\{\begin{array}{l}
\frac{d^{2} u_{1}}{d \tau^{2}}+2 d_{1} \frac{d u_{1}}{d \tau}+f_{1}^{2} u_{1}=m_{1} \frac{d^{2} u_{2}}{d \tau^{2}} \\
\frac{d^{2} u_{2}}{d \tau^{2}}+2 d_{2} \frac{d u_{2}}{d \tau}+f_{2}{ }^{2} u_{2}=m_{2} \frac{d^{2} u_{1}}{d \tau^{2}}
\end{array}\right.
$$

Taking the Laplace Transformation and taking into account the initial conditions:

$$
\begin{array}{ll}
\mathscr{L} u_{1}=U_{1}(0) & \mathscr{L} u_{2}=U_{2}(0) \\
\mathscr{L} \frac{d u_{1}}{d \tau}=S U_{1}(S)-u_{1}(0) & \mathscr{L} \frac{d u_{2}}{d \tau}=S U_{2}(S) \\
\mathscr{L} \frac{d^{2} u_{1}}{d \tau^{2}}=S^{2} U_{1}(S)-S u_{1}(0) & \mathscr{L} \frac{d^{2} u_{2}}{d \tau^{2}}=S^{2} U_{2}(S)
\end{array}
$$

then the equations (11) transform to

$$
\left\{\begin{array}{l}
\left(S^{2}+2 d_{1} S+f_{1}{ }^{2}\right) U_{1}(S)-m_{1} S^{2} U_{2}(S)=\left(S+2 d_{1}\right) u_{1}(0) \\
\left(S^{2}+2 d_{2} S+f_{2}^{2}\right) U_{2}(S)-m_{2} S^{2} U_{1}(S)=-m_{2} S u_{1}(0)
\end{array}\right.
$$

Solving the equations, one gets

$$
U_{1}(S)=\frac{\left(S+2 d_{1}\right)\left(S^{2}+2 d_{2} S+f_{2}^{2}\right)-k^{2} S^{3}}{\left(S^{2}+2 d_{1} S+f_{1}^{2}\right)\left(S^{2}+2 d_{2} S+f_{2}^{2}\right)-k^{2} S^{4}} u_{1}(0)
$$

which can be converted to a partial fraction 


$$
\frac{U_{1}(S)}{u_{1}(0)}=\frac{a_{1} S+b_{1}}{\left(S^{2}+2 d_{1}^{\prime} S+f_{1}^{\prime 2}\right)}+\frac{a_{2} S+b_{2}}{\left(S^{2}+2 d_{2}^{\prime} S+f_{2}^{\prime 2}\right)}
$$

where the parameters $d_{1}, d_{2}, \Omega_{1}, \Omega_{2}^{\prime}, a_{1}, a_{2}, b_{1}$, and $b_{2}$ are to be determined. Taking the inverse Laplace transformation on (15), it is easy to get the final solution:

$$
\frac{u_{1}(\tau)}{u(0)}=A_{1} e^{-d_{1}^{\prime} \tau} \cos \left(f_{a} \tau+\varphi_{a 1}\right)+B_{1} e^{-d_{2}^{\prime} \tau} \cos \left(f_{b} \tau+\varphi_{b 1}\right)
$$

$$
\text { where } \begin{aligned}
& f_{a}=\sqrt{f_{1}^{\prime 2}+d_{1}^{\prime 2}}, A_{1}=a_{1} \sqrt{1+\left(\frac{b_{1}-a_{1} d_{1}^{\prime}}{a_{1} f_{a}}\right)^{2}}, \varphi_{a 1}=\arctan \left(-\frac{b_{1}-a_{1} d_{1}^{\prime}}{a_{1} f_{a}}\right) \\
& f_{b}=\sqrt{f_{2}^{\prime 2}+d_{2}^{\prime 2}}, B_{1}=a_{2} \sqrt{1+\left(\frac{b_{2}-a_{2} d_{2}^{\prime}}{a_{2} f_{b}}\right)^{2}}, \varphi_{b 1}=\arctan \left(-\frac{b_{2}-a_{2} d_{2}^{\prime}}{a_{2} f_{b}}\right)
\end{aligned}
$$

Evidently, the solution consists of two decay harmonics. As mentioned in the preceding section, the composite field is more important, so we write (16) in another form:

$$
\frac{u_{1}(\tau)}{u_{1}(0)}=C_{1} \cos \left(\frac{f_{a}+f_{b}}{2} \tau+\Phi_{1}(\tau)\right)
$$

where

$$
\begin{aligned}
& C_{1}^{2}=A_{1}^{2} e^{-2 d_{1}^{\prime} \tau}+B_{1}^{2} e^{-2 d_{2}^{\prime} \tau}+2 A_{1} B_{1} e^{-\left(d_{1}^{\prime}+d_{2}^{\prime}\right) \tau} \cos \left[\left(f_{a}-f_{b}\right) \tau+\left(\varphi_{a}-\varphi_{b}\right)\right] \\
& \Phi_{1}(\tau)=\arctan \left[\frac{A_{1} e^{-d_{1}^{\prime} \tau}-B_{1} e^{-d_{2}^{\prime} \tau}}{A_{1} e^{-d_{1}^{\prime} \tau}+B_{1} e^{-d_{2}^{\prime} \tau}} \cdot \tan \left(\frac{f_{a}-f_{b}}{2} \tau+\frac{\varphi_{a}-\varphi_{b}}{2}\right)\right]
\end{aligned}
$$

Since $\Phi$ in (17) is also a function of time, the frequency varies from time to time, that is:

$$
\text { freq }=\frac{d}{d \tau}\left(\frac{f_{a}+f_{b}}{2} \tau+\Phi_{1}(\tau)\right)=\frac{f_{a}+f_{b}}{2}+\frac{d \Phi_{1}(\tau)}{d \tau}
$$

Note that the second term gives a significant contribution. Note also all parameters are normalized.

Now the only problem is how to determine the parameters $d_{1}, d_{2},, f_{1}, f_{2}, a_{1}, a_{2}, b_{1}$, and $b_{2}$ in (15) from (14). Since it is hard to get an analytic solution, we calculate numerically via computer code, keeping in mind that reducing the truncation error is essential. A few typicall results are shown below.

Fig. 9 shows the amplitude $C$ of (18). Evidently it is a beat wave formed by two waves. It is conceivable because the decay is slow, the two waves do form a beat wave except the occurrence of decay, which results in a single frequency eventually.

Fig. 10 shows the frequency variation expressed in (19). It shows that the frequency does vary periodically. The original frequency of primary loop is $f_{10}=1$. Fig. 10 (b) shows that the oscillation becomes slower when the frequencies are closer together. 

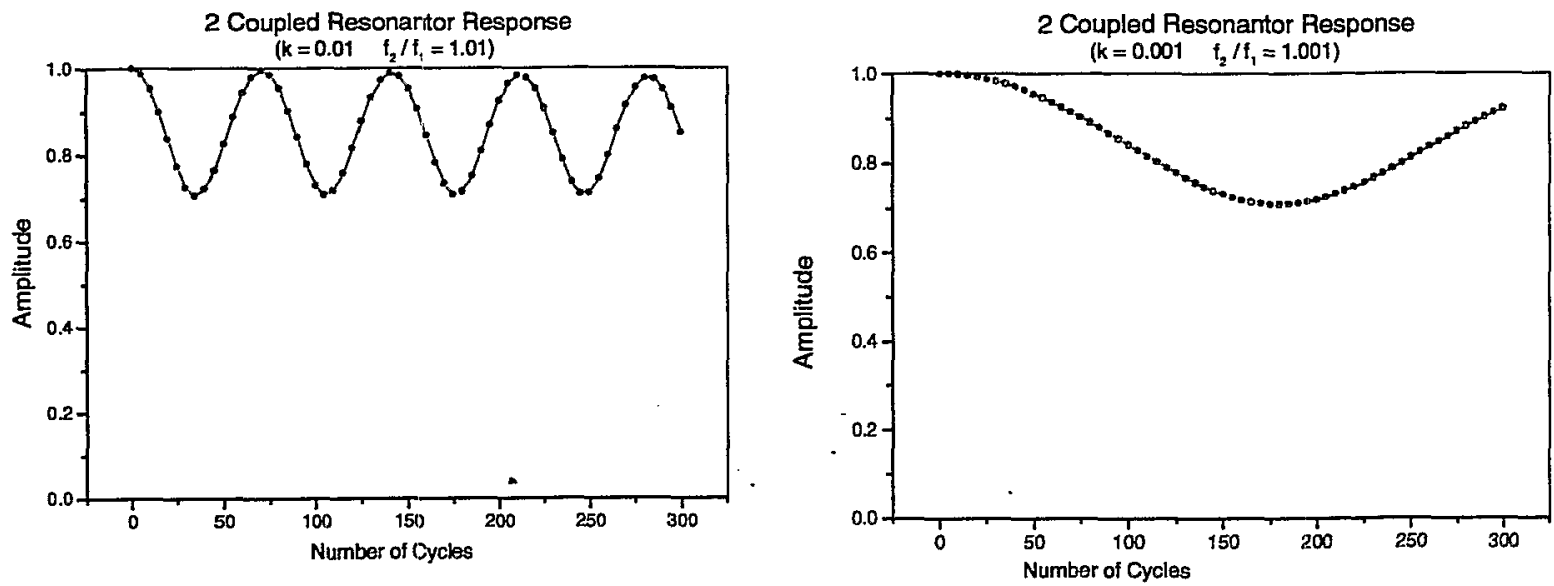

Fig. 9 The two waves form a decaying beat wave with
(a) $f_{2} / f_{1}=1.01, k=0.01$ and
(b) $f_{2} / f_{I}=1.002, k=0.002$
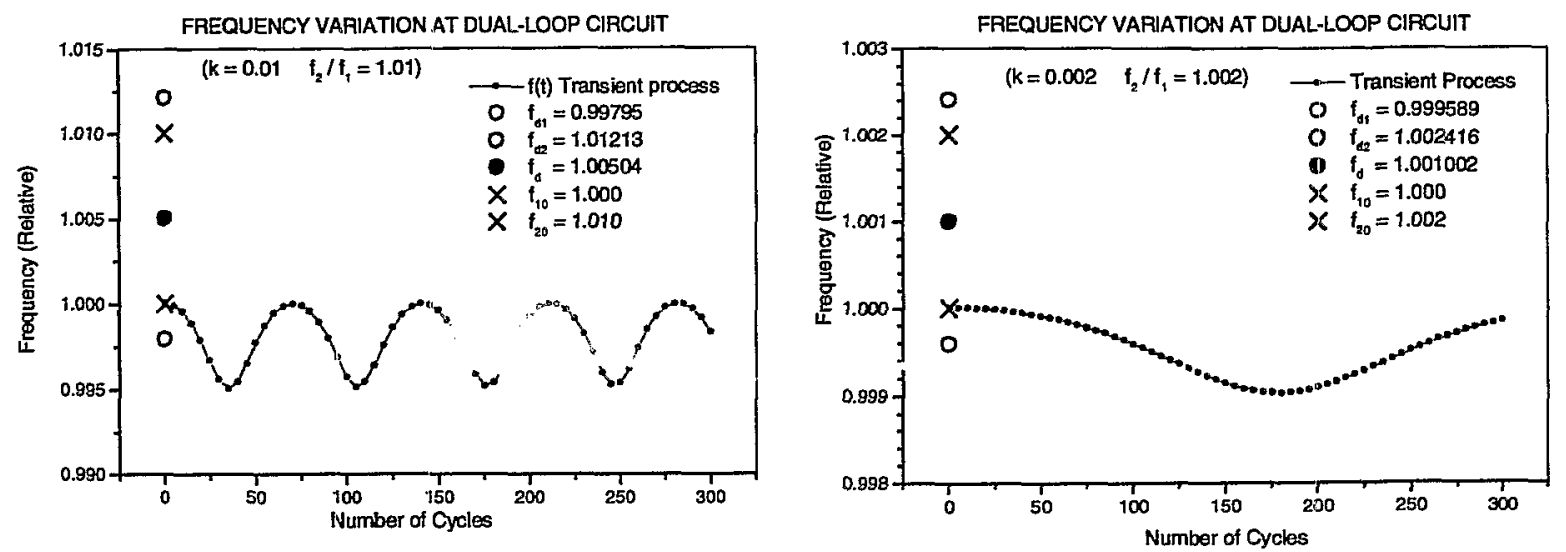

Fig. 10 The frequency variation with different parameters with

(a) $f_{2} / f_{1}=1.01, k=0.01$ and (b) $f_{2} / f_{1}=1.002, k=0.002$
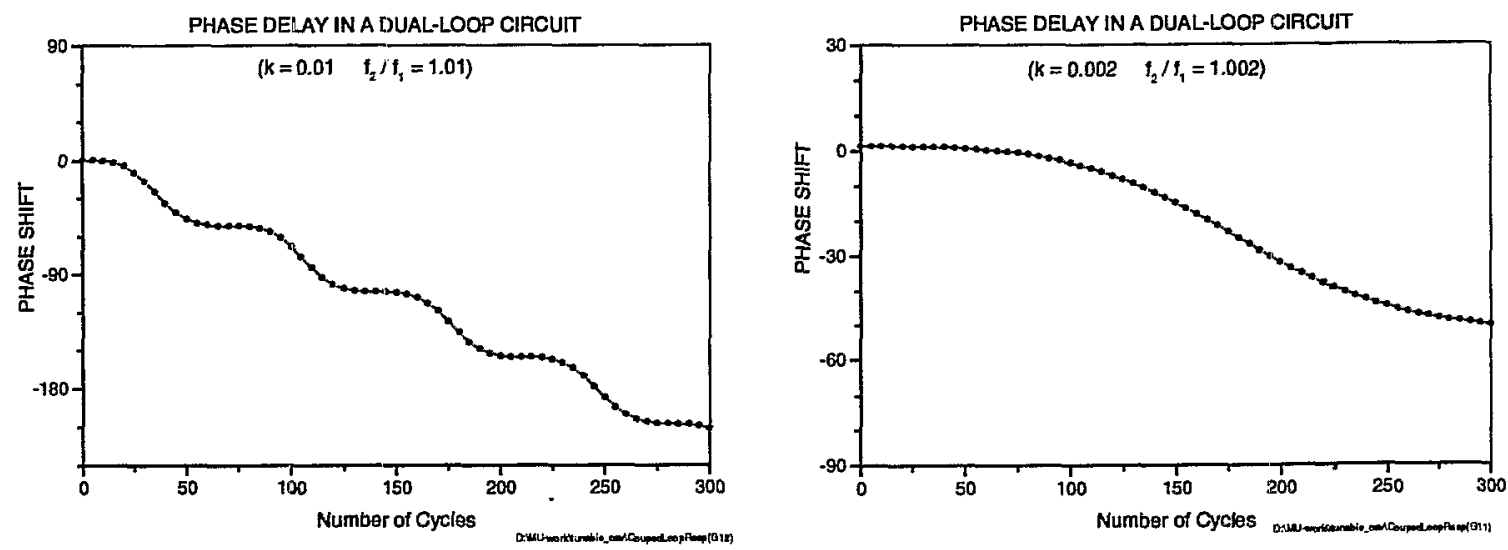

Fig.11. The phase delay with different parameters with

(a) $f_{2} / f_{1}=1.01, k=0.01$ and (b) $f_{2} / f_{l}=1.002, k=0.002$ 
Intuitively, we presume the composite wave will have its frequency shifted to the middle of the two original resonant frequencies before being coupled. Finally we found this is not the case. The frequency oscillates around $f_{d l}$, but does not tend towards $f_{2}$. Instead, it approaches $f_{d I}$ after a significant decay, when the $f_{d l}$ component becomes dominant. More detailed analyses are given in the Appendix.

In the above figure, the simulation covers only 300 cycles, implying only 1.5 microsecond for $200 \mathrm{MHz}$. This time-span, $1.5 \mu \mathrm{S}$, is much smaller than that required for a single frequency becorning dominated.

Nevertheless, as mentioned before, we are concerned about the phase change that occurs when the beam comes back in the next cycle. Fig. 11 shows the corresponding phase delay of Fig. 10. With the particular parameters, the delay is over 180 degrees in 1.5 $\mu s$. The phase change/delay is defined as the difference of the phase of (17) and the phase if the 2 nd loop has not been introduced. That is:

$$
\Phi_{d}=\frac{f_{a}+f_{b}}{2} \tau+\Phi_{1}(\tau)-f_{1} \tau
$$

If one choose the 2 nd loop frequency lower than the 1 st one, e.g. $f_{2} / f_{1}=0.99$, then all results will be reversed. The phase will be advancing, instead of delayed.

The above analysis reveals that introducing a 2 nd resonant loop to change the main frequency is not effective. But changing the phase may work out. We'll address that later.

\section{A SIMPLE CAVITY SCHEME}

As discussed above the dual-loop scheme does not give a good solution. We now consider a simple cavity with the deformed shape shown in Fig. 12. The wall on the magnetic region is deformed to form a "cave", in which the flux can be changed for tuning.

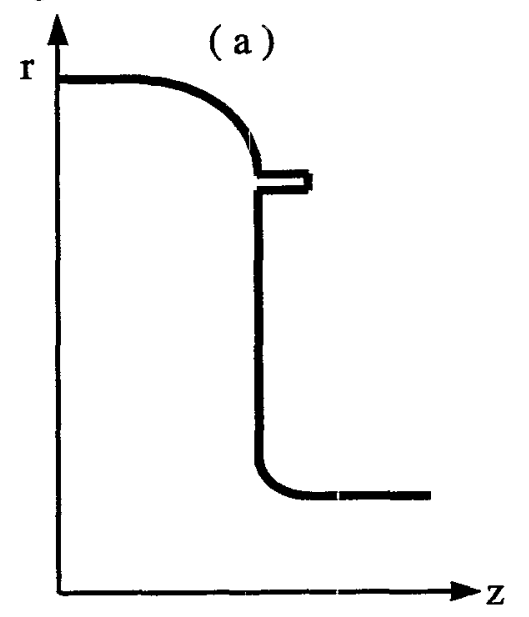

(b)

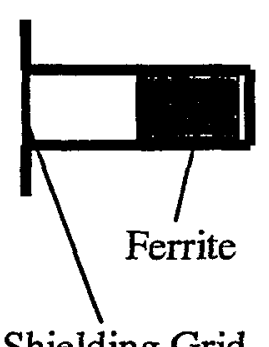

Shielding Grid (c)

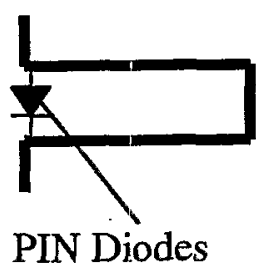

PIN Diodes

Fig. 12 Deformed cavity with a "cave" region ( a ), which is enlarged in the right view. It is tunable by means of ferrite ( $b$ ), or PIN diodes ( c ).

Fig.12 (b) and (c) show an expanded view of the cave region. It shows two options to change the flux by ferrite and PIN diodes respectively. The ferrite changes the 
permeability by means of its bias. PIN diodes form switches to open or close the cave region.

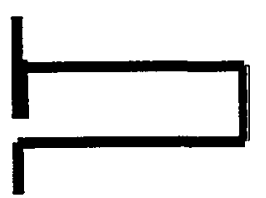

Fig. 13 The magnetic flux pattern with the grid not shorted (shown above).

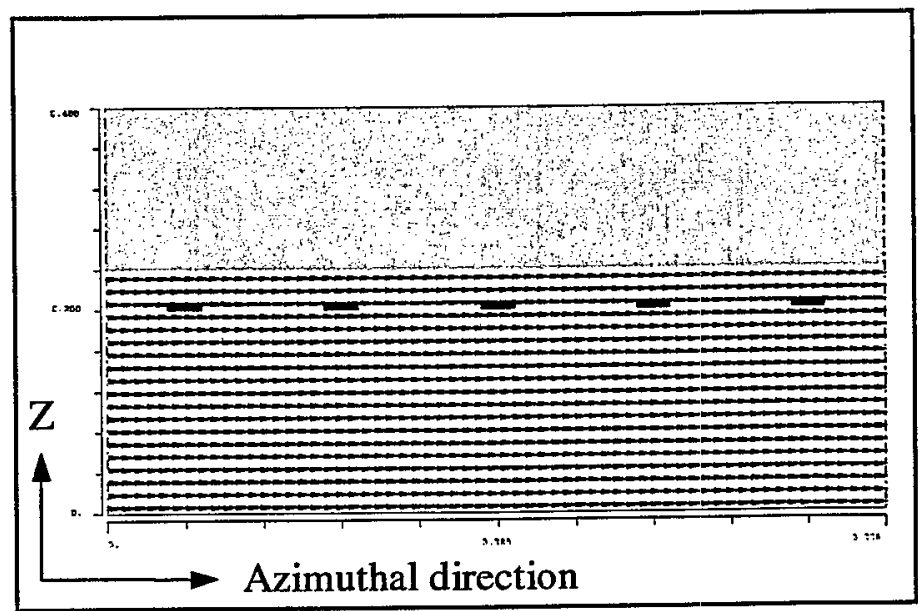

Figs. 13 and 14 show the flux pattern in two cases resulting from MAFIA simulation. Note the horizontal axis is along the azimuthal direction. In Fig.13, the grid is "open", and the flux inside the cave region is parallel with the outside flux. When the grid is "shorted", Fig.14, part of the flux is screened off. These simulate the two PIN states -open / closed. In this simulation, the cross-section of the cave region is $2 \times 5 \mathrm{~cm}^{2}$ of a $200 \mathrm{MHz}$ cavity, the frequency change is $0.82 \mathrm{MHz}$ or $0.4 \%$. Obviously, changing the cross section will change the tuning rate. A tuning range of $1 \%$ is possible.

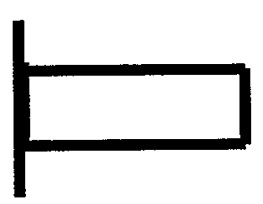

Fig.14 The magnetic flux pattern with the grid shorted (shown above).

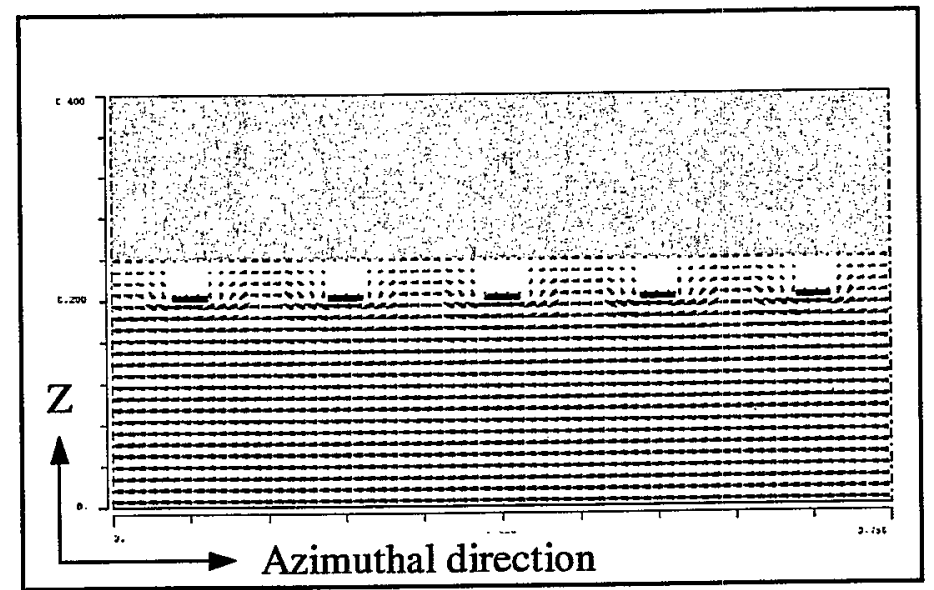

For the ferrite option the flux is very intense in the high gradient cavity. In order to weaken it to make the ferrite tolerable, one possible measure is to partially screen the flux by a grid on the opening of the cave region. Fig 14 also shows the effect of a grid. The flux density is weakened in average, but it is nonuniform. At some points the density is still too high for ferrite, unless the grid density is further increased. The latter implies more loss and less tuning range.

Next, we'll investigate the ferrite and PIN diodes.

\section{FERRITE AND OTHER MAGNETIC MATERIAL}

Ferrite has been widely used for tuning cavities in accelerators. Some examples are 
as follows.

The BNL light source used ferrite in a $52 \mathrm{MHz}$ cavity to compensate the synchrotron radiation with a tunability of $78 \mathrm{kHz}^{[2]}$. In the SSC LEB ring, a cavity was designed with tuning range of 47.5 to $59.8 \mathrm{MHz}$ and a cavity voltage of $127.5 \mathrm{kV}$ each ${ }^{[3]}$. Fermi Lab has developed a cavity of $159 \mathrm{MHz}$ tunable of $60 \mathrm{kHz}$ by an Iron-Yttrium-Garnet ferrite with a gap voltage of $300 \mathrm{kV}{ }^{[4]}$. Indiana University developed a wide tuning range cavity of 1.2 to $10 \mathrm{MHz}$ employing ferrite rings made of Phillips $8 \mathrm{C} 12$. But it was operated with low level driver of 300 watt and cavity voltage of $500 \mathrm{~V}^{[5]}$. TRIUMF developed a cavity with tuning range of 46.1 to $60.6 \mathrm{MHz}$ and gap voltage of $62.5 \mathrm{kV}{ }^{[6]}$. Los Alamos also developed a cavity with a tuning range of 50 to $60 \mathrm{MHz}$ and gap voltage of $130-140$ $\mathrm{kV}^{[7]}$.

Our question is whether a ferrite is able to operate at a higher frequency (say 200 $\mathrm{MHz}$ ) and with strong fields, where the cavity gap voltage is on the order of a mega-volt. There are four factors are of major concern for ferrite materials.

1) The upper limit of frequency, beyond which the $\mu$ drops and the loss increases rapidly.

2) The maximum tolerable RF magnetic flux, which determines the energy density and the field intensity. Most ferrite is limited to 100 gauss.

3) The range of the relative permeability, which determines the tuning capability. For parallel bias, the $\mu_{\mathrm{rf}}$ can be higher than $180^{[8]}$, even 800 in low level ${ }^{[5]}$, but a parallel bias also accompanies a high loss. For perpendicular bias the loss is lower, but its $\mu_{\mathrm{rf}}$ may be as low as 1.2 to $4.5^{[7]}$.

4) The manageable average power dissipation, which determines the applicable power and the volume of material. It depends not only on the material itself and its Curie temperature, but also on the cooling method. The dissipation should be on the order of 10 $\mathrm{W} / \mathrm{cm}^{3}$ or less.

These factors are very complex and often related each other. In addition, there are lots of engineering concerns, like the layout of the bias coil and the orientation, sparking, conditioning, cooling, the feasibility of size, economy etc.

As to the requirement of fast tuning, a special concern arises. One should avoid the metallic screen effect of the cavity envelope, of which the ferrite is inside, but the bias coil is outside. The eddy current along the flux loop must be taken into serious consideration. Presumably, a tuning speed of one microsecond scale will encounter big difficulties.

According to the above data, although ferrite was widely used in many labs, the gap voltages were all moderate and the frequencies were low. The Fermi Lab's example shows the highest frequency of $159 \mathrm{MHz}$ and the highest voltage of $300 \mathrm{kV}$. But, its tuning range is very narrow, only $0.04 \%$. Narrow tuning range implies a small percentage of energy going into the ferrite.

As is well known, ferrite is a nonlinear and anisotropic material. Not only is its B-H relationship curved and history dependent, but also its permeability is a tensor. Roughly speaking, the permeability is $\mu=\mathrm{B} / \mathrm{H}$ in the dc case, and $\mu_{\mathrm{rf}}=\Delta \mathrm{B} / \Delta \mathrm{H}$ at radio frequency. 
The latter is strongly dependent on the dc bias magnetic field, which makes tuning possible. The $\mu_{\mathrm{rf}}$ also depends on the angle between the bias field and rf field. There are two common cases, with the rf magnetic field perpendicular to the dc bias field and parallel each other. For perpendicular bias case, the $\mu_{\mathrm{rf}}$ can be expressed approximately as ${ }^{[9]}$

$$
\mu_{r f}=1+\frac{4 \pi M_{s}}{H}
$$

where $4 \pi M_{s}$ is the saturation magnetization. Normally $\mu_{\mathrm{rf}}$ is much less in the perpendicular case than in the parallel. But its $Q$ is much higher. This makes it possible to apply in a relatively high field. Therefore it has been widely used recently, including the above-mentioned examples. ${ }^{[7]}$

The area of the hysteresis loop determines the major loss per cycle. Therefore the total loss is proportional to the frequency. The shape of hysteresis loop is dependent on the dc bias and the rf amplitude. Near saturation the loop is approximately a straight line. Then the loss reaches its minimum and the $\mathrm{Q}$ a maximum. But, its $\mu_{\mathrm{rf}}$ also reaches its minimum.

The $\mu \mathrm{Qf}$-product is a figure of merit of ferrite. Therefore, for higher frequency one must sacrifice $\mu$ or $Q$. Besides, when the power increases, or the rf magnetic flux intensity increases, the $\mu \mathrm{Qf}$-product will drop in most cases, making things more serious. Note here $\mu$ is $\mu_{\mathrm{rf}}$, and $\mathrm{Q}$ is the magnetic $\mathrm{Q}$ of the ferrite. It is defined as

$$
\mathrm{Q}=\omega \mathrm{W}_{\mathrm{m}} / \mathrm{P}_{\mathrm{m}}
$$

where $W_{m}$ is the pealk energy stored inside the ferrite and $P_{m}$ the dissipated power. Similarly, an electric Q may be defined, but it has little importance for tuning.

In recent years, a met-glass-like material called Finemet was developed in Japan ${ }^{[8]}$. Finemet is in the form of a tape, which can be wound into a large core. The advantage is that it can hold up to 2000 gauss of magnetic flux intensity, while ferrite is often limited to only $100 \sim 200$ gauss. But Finemet is very lossy and can only work at low frequency, say several MHz. In Fermi Lab's example, it works with $2 \mathrm{MHz}$ only. ${ }^{[8]}$

Recently, KEK reported a new ferrite called TDK SY20 ${ }^{[10]}$. Its $\mu$ Qf-product reaches $6 \times 10^{10}$ at low level and can operate at $B_{\mathrm{rf}}$ about 300 Gauss. But, it is suitable only for low frequency, say several MHz.

Despite some advantages, all these new materials are unlikely to be workable at 200 MHz.

\section{PIN DIODES}

PIN diodes have been widely used for microwave switches or attenuators. In contrast to ferrite, the PIN diode is suitable for high frequency. When the PIN diode is forward biased, holes and electrons are injected into the I-region. This charge does not recombine instantaneously, but has a finite lifetime $\tau$. If a dc current of $I_{f}$ is forward biased, the stored charge in the I-region is

$$
Q_{s}=I_{f} \tau
$$


If in the mean time there is RF current $I_{r f}$ passing through it, it carries out an RF charge $Q_{r}$, which is the integral of $I_{r f}$, or

$$
Q_{r f}=I_{r f} / \omega \text {. }
$$

Providing $Q_{f f} \ll Q_{S}$, the PIN diode behaves like a conductor. Evidently, the higher the frequency, the more RF current can be carried over. Therefore, usually there exists a low frequency limit. Of course, a high frequency limit also exists due to other restrictions.

If the diode is reverse biased, there is no stored charge in the I-region and the device behaves like a capacitance. It is the difference in performance between the forward and reverse bias states upon which the switch operation relies. Of course, neither "open" nor "close" is perfect. There exists resistance, that causes heat, which will restrict the applicable RF power.

To my knowledge after investigation, up to now, the commercial available highest power PIN diode is UM4300 series produced by Microsemi Corp. Its peak power is $500 \mathrm{~kW}$ in a condition of single pulse with a duration of $1 \mu \mathrm{s}$. At $200 \mathrm{MHz}$, the performance is nearly linear. i.e. if the duration is $10 \mu \mathrm{s}$, then the peak power is 10 times less, or $50 \mathrm{~kW}$. The voltage rating is $1000 \mathrm{~V}$. For continuous operation the power dissipation rating is 20 Watts.

Another model HUM3002 has a maximum reverse voltage of 3000V, and its Power dissipation is $75 \mathrm{~W}$.

The engineering feasibility needs more cautiousness. Power dissipation depends on many parameters and is a major limiting factor in determining the maximum RF power level that the PIN diode switch can control without overheating.

\section{PHASE TUNING}

As mentioned at the beginning, changing frequency and changing phase can be equivalent for the FFAG. Table 1 lists an example that requires a frequency shift of $0.23 \%$, while requires a phase shift of 648 degree. Intuitively, phase tuning is more difficult than frequency tuning. However, this may not be always true.

Let's assume a cavity (as shown in Fig. 12) has two states as changing the bias of either a ferrite or a set of PIN diodes. Two states correspond to two different eigenfrequencies. By changing the width of the bias pulse, one can easily change the phase shift between two successive bunches.

Fig. 15 shows the principle. The phase is an integral of the frequency as follows.

$$
\begin{aligned}
& \Phi=\int 2 \pi f \cdot d t \\
& \Delta \Phi=2 \pi \int_{w} \Delta f \cdot d t=2 \pi\left(f_{2}-f_{1}\right) \cdot w
\end{aligned}
$$

So, changing the pulse width $w$ will change the $\Delta \Phi$. This scheme has the following advantages. 

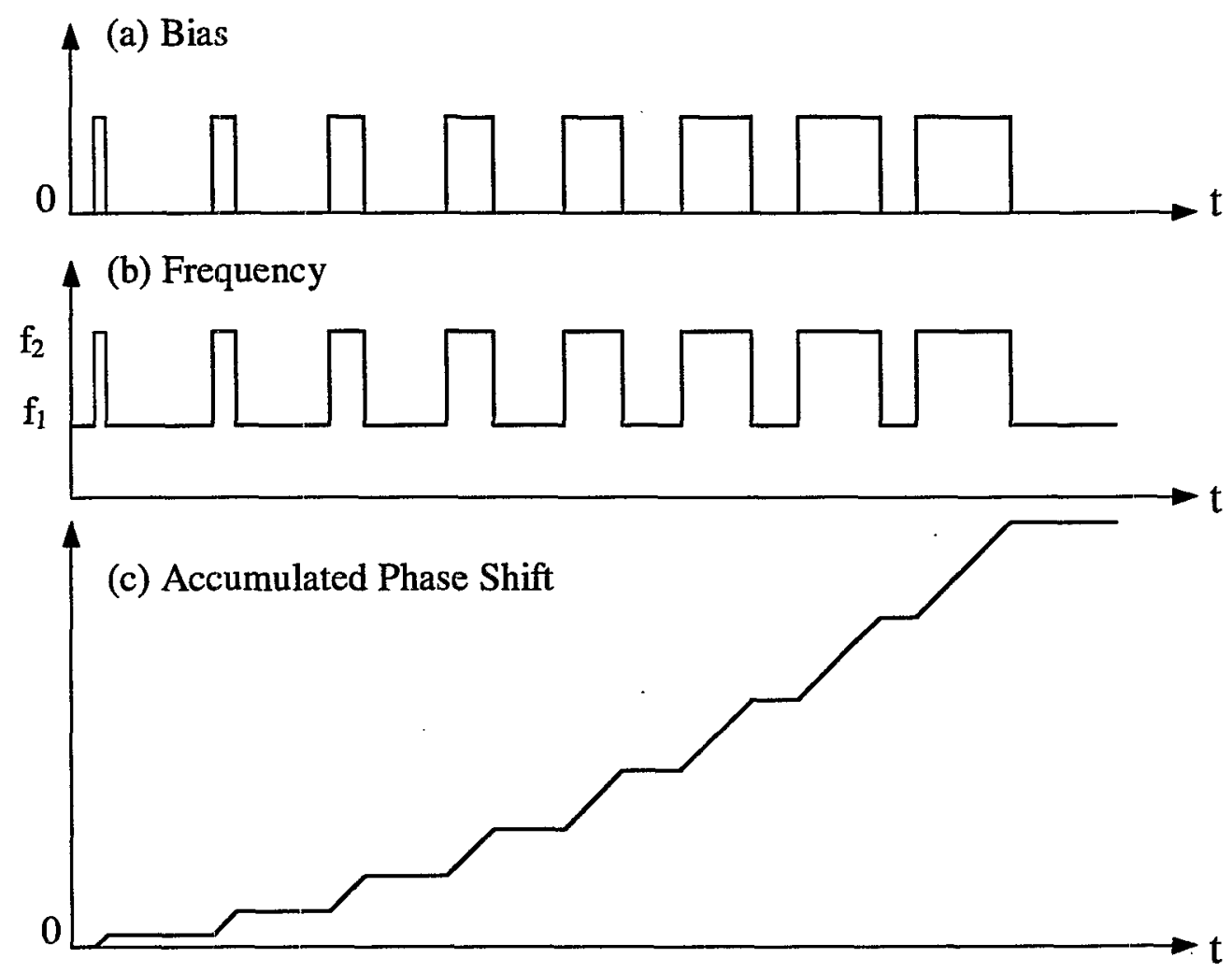

(d) Bunches

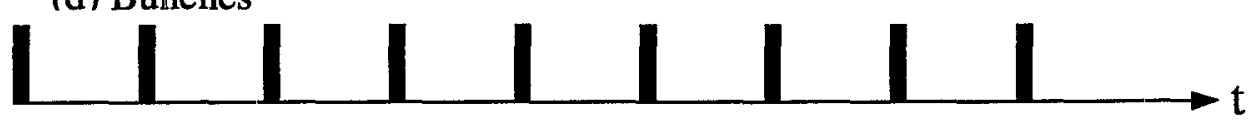

Fig. 15 Phase tuning by modulation of the bias pulse width.

1. There are only two states of bias, making it relatively easy to minimize the loss of the device, i.e. the loss of the ferrite or the PIN diodes.

2. Only one parameter is needed to be controlled, i.e. $w$. For frequency tuning, one has to control both the frequency and its phase.

3. The control can be programmable. In practice, trimming is always necessary and this scheme is easy to compensate system error.

4. The width control is flexible. The relationship between revolution time and the momentum (Fig.1) can be of any shape regardless of nonlinearity.

5. If the required maximum phase shift between two successive bunches is moderate, the required frequency difference between two states (i.e. $f_{2}-f_{l}$ ) is about the same in both frequency tuning and phase tuning. But, if the required phase difference is large, the phase tuning will require less frequency difference, because in any case, the phase shift of $2 \pi$ is enough. This, according to (24), requires a frequency difference of $f_{2}-f_{1}=1 / \tau$. For frequency tuning, according to (6), it is proportional to $\Delta \tau$.

However, problems remain. The power handling capability of the device is the same. Its availability is doubtful. For PIN diode, rapid switch on and off is impossible in a time scale of 1 microsecond or less. Rapidly changing the bias of the ferrite is also a challenge. So new $R$ \& $D$ is a must. 


\section{ENERGY ISSUE}

Energy plays an essential role in any physical system. For a tuning system one must control a certain amount of energy, which is proportional to the whole energy stored in this system. Let's consider a simple resonant circuit to see how much power is needed to be controlled.

Fig.16 shows an equivalent circuit of a simple cavity. As is well known

$$
Q=\omega W / P
$$

Here $W$ is the peak energy stored in the capacitance $C$ or the inductance $L$, while $P$ is the power dissipated on the wall or the resistance $R$ in the equivalent circuit. It is also the drive power from the source. The reactive power in $L$ or $C$ is:

$$
P_{L}=P_{C}=\omega C V^{2}=2 \omega W=2 P Q
$$

If one wants to change frequency by changing the inductance, the increment of the inductance should be:

$$
\Delta L=L \times 2 \Delta f / f
$$

The power stored in the $\Delta L$ is what has to be

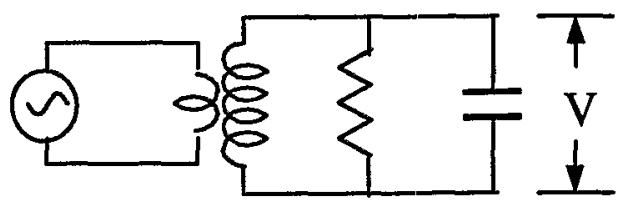

Fig.16 The equivalent circuit of a simple cavity controlled, and is:

$$
\Delta P=P_{L} \times 2 \Delta f / f=2 \omega W \times 2 \Delta f / f=8 \pi W \times \Delta f
$$

Note that, from (26), the reactive power $P_{L}$ is $2 Q$ times larger than the driver power, which is for compensating the wall loss only. Formulae (26) and (28) also show that, if the gap voltage is specified, that the energy $W$ is also determined, the controlled power as well as the reactive power does not depend on $Q$.

Table 3 shows the numerical results of a SUPERFISH run, and those deducted from the above equations. The cavity has a normal bell shape and the axial length is $0.6 \mathrm{~m}$ with frequency near $200 \mathrm{MHz}$. The last two rows list the controlled power with the tuning ranges of $0.1 \%$ and $1 \%$ respectively. Also listed is the ratio of voltage to the Kilpatrick criteria.

Table 3

\begin{tabular}{|c|c|c|c|c|c|}
\hline $\mathrm{E}_{0}$ (gradient)* & $\mathrm{MV} / \mathrm{m}$ & 1 & 1.67 & 2 & 10 \\
\hline Gap Voltage & $\mathrm{MV}$ & 0.60 & 1.0 & 1.2 & 6.0 \\
\hline Kilpatrick ratio & & 0.12 & 0.20 & 0.24 & 1.2 \\
\hline $\mathrm{W}$ (stored energy) & Joule & 1.205 & 3.347 & 4.820 & 120.5 \\
\hline $\mathrm{P}$ (wall loss) & $\mathrm{kW}$ & 26.68 & 74.1 & 106.7 & 2688 \\
\hline $\mathrm{P}($ reactive) & $\mathrm{MW}$ & 2930 & 8139 & 11726 & 293150 \\
\hline$\Delta \mathrm{P}(\Delta \mathrm{f} / \mathrm{f}=0.1 \%)$ & $\mathrm{MW}$ & 5.86 & 16.3 & 23.4 & 586 \\
\hline$\Delta \mathrm{P}(\Delta \mathrm{f} / \mathrm{f}=1 \%)$ & $\mathrm{MW}$ & 58.6 & 163 & 234 & 5860 \\
\hline
\end{tabular}

* SUPERFISH gives normalized results of $1 \mathrm{MV} / \mathrm{m}$, the rest are those scaled up. 
The needed controlled power as well as the stored energy increase rapidly with voltage squared. So, if the voltage per cavity is $6 \mathrm{MV}$, which is desirable, the Kilpatrick ratio is quite moderate, but the $\Delta \mathrm{P}$ is way too high.

To estimate the specifications of the required devices, Table 4 gives the voltage and current at the cave region in the above example.

Table 4

\begin{tabular}{|c|c|c|c|}
\hline Gap Voltage & $\mathrm{MV}$ & 0.6 & 6 \\
\hline Cave Voltage & $\mathrm{kV}$ & 4.5 & 45 \\
\hline $\mathrm{H}_{\max }$ at cave & $\mathrm{A} / \mathrm{m}$ & 2419 & 24190 \\
\hline Total current & $\mathrm{A}$ & 3040 & 30400 \\
\hline
\end{tabular}

Obviously, neither a ferrite nor a PIN diode can meet the requirements. Even if one may design an alternative structure, of which the required voltage can be significantly reduced, it still requires thousands of PIN diodes in parallel. That is not practical, unless one can develop much large PIN diodes.

\section{SUMMARY}

The rapid tuning cavity with high power has been investigated. So far no satisfied solution is reached yet. Nevertheless, we learnt the following.

1. A coupled two loops system has two eigenfrequencies, which may split sufficiently. But, as free oscillation occurs, it forms a beat wave-like oscillation, the frequency of the composite wave has much less deviation.

2. A siagle cavity, which is equivalent to a single loop with a single eigenfrequency (ignoring high order modes here), was considered. The possible structure with ferrite or PIN diodes was studied. The available ferrite and PIN diodes were also inve ated. Unfortunately, neither ferrite nor PIN diode, to state-of-the-arts, can meet the requirements in the high power in consideration.

3. The phase tuning scheme was studied. It has some advantages and may be applied in some special cases. The devices are also a restriction for applications.

4. The energy is a vital factor. It reveals that there is still a long way to go to reach a high power rapid tuning.

\section{ACKNOWLEDGEMENT}

Many thanks to $\mathbf{S}$. Berg for his raising this issue and his review and valuable comments of this manuscript. Thanks also to CAP group, who gave valuable discussions continuously.

\section{References}

[1] S.J. Scott, private communication

[2] E. Pivit, S. M. Hanna and J. Keane, "Fast ferrite tuner for the BNL synchrotron light 
source", PAC91.

[3] P.Coleman et al. "Status of SSC LEB RF cavity", PAC93, p.824, and "Test results on the SSC low energy booster RF cavity", EPAC94.

[4] J. Dey, C. M. Bhat and D. Wildman, "The high level RF system for transition crossing without RF focusing in the main ring at Fermilab", PAC93, p.1223

[5] A. Pei et al. "The Indiana University cooler injection synchrotron RF cavity", Linac Conf. 2000.

[6] R. L. Poirier, " Perpendicular biased ferrite tuned cavity", PAC93. p.753

[7] W. R. Smythe and T. G. Brophy, "RF cavities with transversely biased ferrite tuning", IEEE Trans. on Nuclear Science, NS-32, no.5, p.2951, 1985.

[8] K. Y. Ng and Z. B. Qian, "Finemet versus ferrtie -- pros and cons", PAC99, p.872.

[9] W. R. Smythe, "Reclucing ferrite tuner power loss by bias field rotation", IEEE Trans. on Nuclear Science, NS-30, No.4. p.2173, 1983.

[10] Kiyomi Koba, "Low frequency RF system for neutrino factory", NuFact00 Workshop Monterey, California, May 22-26, 2000

[11] C. Ohmori et al. (KEK), "High field gradient cavity loaded with magnetic alloys for synchrotrons", Proceedings of PAC99, p.413, 1999. 


\section{APPENDIX \\ The frequency of a beat wave}

As is well known two waves with their frequencies very close each other form a beat wave. Intuitively, the frequency of the composite wave is equal to or close to their average. However, it may not be always true.

Let's consider the following simple case

$$
u(t)=A \cos \left(\omega_{1} t\right)+B \cos \left(\omega_{2} t\right)
$$

It can be converted to:

$$
u(t)=C \cos \Psi(t)
$$

where

$$
\begin{aligned}
& C^{2}=A^{2}+B^{2}+2 A B \cos \left(\omega_{1}-\omega_{2}\right) t \\
& \Psi(t)=\frac{\omega_{1}+\omega_{2}}{2} t+\Phi(t) . \\
& \Phi(t)=\arctan \left(\frac{A-B}{A+B} \tan \frac{\omega_{1}-\omega_{2}}{2} t\right)
\end{aligned}
$$

$C$ is the amplitude or the envelope, and $\Psi$ is the total phase angle. Both are functions of time,

In a special case, $A=B, \Phi \equiv 0$, the angular frequency of the composite wave is

$$
\omega_{0}=\frac{\omega_{1}+\omega_{2}}{2}
$$

Only in this case, that two waves have equal amplitudes, the frequency of the composite wave is equal to their average. But, if $A \neq B$, the case is much complex.

Since $\Phi$ is a function of time, the meaning of frequency is somewhat ambiguous. Generally, one can define the frequency to be:

$$
\omega=\frac{d \Psi}{d t}=\frac{\omega_{1}+\omega_{2}}{2}+\frac{d \Phi}{d t}
$$

One may also argue if this expression is precise. Another definition may be as follows. Since the wave is sinusoidal, any phase change of $2 \pi$ can be regarded as one period, of which the reciprocal refers to the frequency. If

$$
\Psi(t+T)-\Psi(t)=2 \pi
$$

then $T$ is the period. The frequency and angular frequency are

$$
f=\frac{1}{T}, \quad \text { and } \quad \omega=\frac{2 \pi}{T}
$$

According to (A4) and (A5), one can find the time function of $T$ and $f$. Rewrite (A5) and (A4) and substitute (A6):

$$
\begin{aligned}
\Phi & =\arctan \left(A_{p} \tan \frac{1}{2} \Delta \omega t\right) \\
\Psi(t) & =\omega_{0} t+\Phi(t) \\
& =\omega_{0} t+\arctan \left(A_{p} \tan \frac{1}{2} \Delta \omega t\right)
\end{aligned}
$$


where $\quad A_{p}=\frac{A-B}{A+B}$ and $\Delta \omega=\omega_{1}-\omega_{2}$

We assume that the two frequencies are very close, that

$$
\Delta \omega<<\omega_{0}
$$

further define

$$
\begin{aligned}
& T_{0}=\frac{2 \pi}{\omega_{0}} \\
& T=T_{0}+\delta \\
& \theta=\frac{1}{2} \Delta \omega \cdot t, \quad \Delta \theta=\frac{1}{2} \Delta \omega \cdot T
\end{aligned}
$$

where $\delta$ is a perturbation term of the period. From (A11),

$$
\Psi(t+T)=\omega_{0} \cdot(t+T)+\arctan \left(A_{p} \tan \frac{1}{2} \Delta \omega(t+T)\right)
$$

From (A13) to (A16),

$$
\Delta \theta=\frac{1}{2} \Delta \omega \cdot T=\frac{1}{2} \Delta \omega \cdot\left(T_{0}+\delta\right) \ll 1
$$

then

$$
\begin{aligned}
& \arctan \left(A_{p} \tan \frac{1}{2} \Delta \omega(t+T)\right)=\arctan \left(A_{p} \tan (\theta+\Delta \theta)\right) \\
& =\arctan \left(A_{p} \tan \theta\right)+\Delta \theta \frac{d}{d \theta} \arctan \left(A_{p} \tan \theta\right) \\
& =\arctan \left(A_{p} \tan \frac{1}{2} \Delta \omega \cdot t\right)+T \cdot \frac{d}{d t} \arctan \left(A_{p} \tan \frac{1}{2} \Delta \omega \cdot t\right) \\
& =\Phi(t)+T \cdot \frac{d \Phi(t)}{d t}
\end{aligned}
$$

Thus from (A18) an (All1) we get

$$
\Psi(t+T)-\Psi(t)=\omega_{0} \cdot T+T \frac{d \Phi(t)}{d t}
$$

Substituting into (A8)

$$
\omega_{0} T+T \frac{d \Phi(t)}{d t}=2 \pi
$$

From (A9) we obtain:

$$
\omega=\frac{2 \pi}{T}=\omega_{0}+\frac{d \Phi(t)}{d t}
$$

Now we get the same expression as (A7) and justify its precision.

Next, let's calculate the frequency variation.

$$
\begin{aligned}
\frac{d \Phi}{d t} & =\frac{d}{d t} \arctan \left(A_{p} \tan \theta\right)=\frac{1}{1+\left(A_{p} \tan \theta\right)^{2}} \frac{d}{d t}\left(A_{p} \tan \theta\right) \\
& =\frac{A_{p} \cdot}{\cos ^{2} \theta+A_{p}^{2} \sin ^{2} \theta} \frac{d \theta}{d t} \\
\frac{d \Phi}{d t} & =\frac{\frac{1}{2} \Delta \omega \cdot A_{p}}{\cos ^{2} \theta+A_{p}^{2} \sin ^{2} \theta}
\end{aligned}
$$


Check three special cases, we have:

$$
\begin{array}{llll}
A=B, & A_{p}=0, & d \Phi / d t=0, & \omega=\omega_{0} \\
B=0, & A_{p}=1, & d \Phi / d t=\Delta \omega / 2, & \omega=\omega_{1} \\
A=0, & A_{p}=-1, & d \Phi / d t=-\Delta \omega / 2, & \omega=\omega_{2}
\end{array}
$$

Surely they are all expected. In general, $A \neq B, d \Phi / d t$ is not constant, but varies between its maximum and minimum. Assuming $A>B$, and disregarding the sign for time being, it does not loss its generality. From (A23), its maximum and minimum are:

$$
\begin{aligned}
& \left.\frac{d \Phi}{d t}\right|_{\max }=\frac{1}{2} \Delta \omega \cdot \frac{1}{A_{p}} \\
& \left.\frac{d \Phi}{d t}\right|_{\min }=\frac{1}{2} \Delta \omega \cdot A_{p}
\end{aligned}
$$

Therefore, the frequency expressed by (A22) will oscillate between these two limits. For example, assuming $B=A / 3, A_{p}=0.5$, the maximum and minimunn frequencies are:

$$
\begin{aligned}
& \omega_{\max }=\omega_{0}+\Delta \omega=\omega_{1}+\Delta \omega / 2 \\
& \omega_{\min }=\omega_{0}+\Delta \omega / 4=\omega_{1}-\Delta \omega / 4
\end{aligned}
$$

It is clear that the frequency is swinging about $\omega_{1}$. Suppose $\omega_{1}>\omega_{2}$, or $\Delta \omega>0$, then $\omega_{\min }$ is in between $\omega_{1}$ and $\omega_{2}$, but $\omega_{\max }$ is larger than both $\omega_{1}$ and $\omega_{2}$. Similarly, if $\omega_{1}<\omega_{2}$ the frequencies is also oscillating about $\omega_{1}$. When $B>A$, the oscillation is about $\omega_{2}$. Fig.A1 illustrates the phenomenon with $B=0.5 A$.
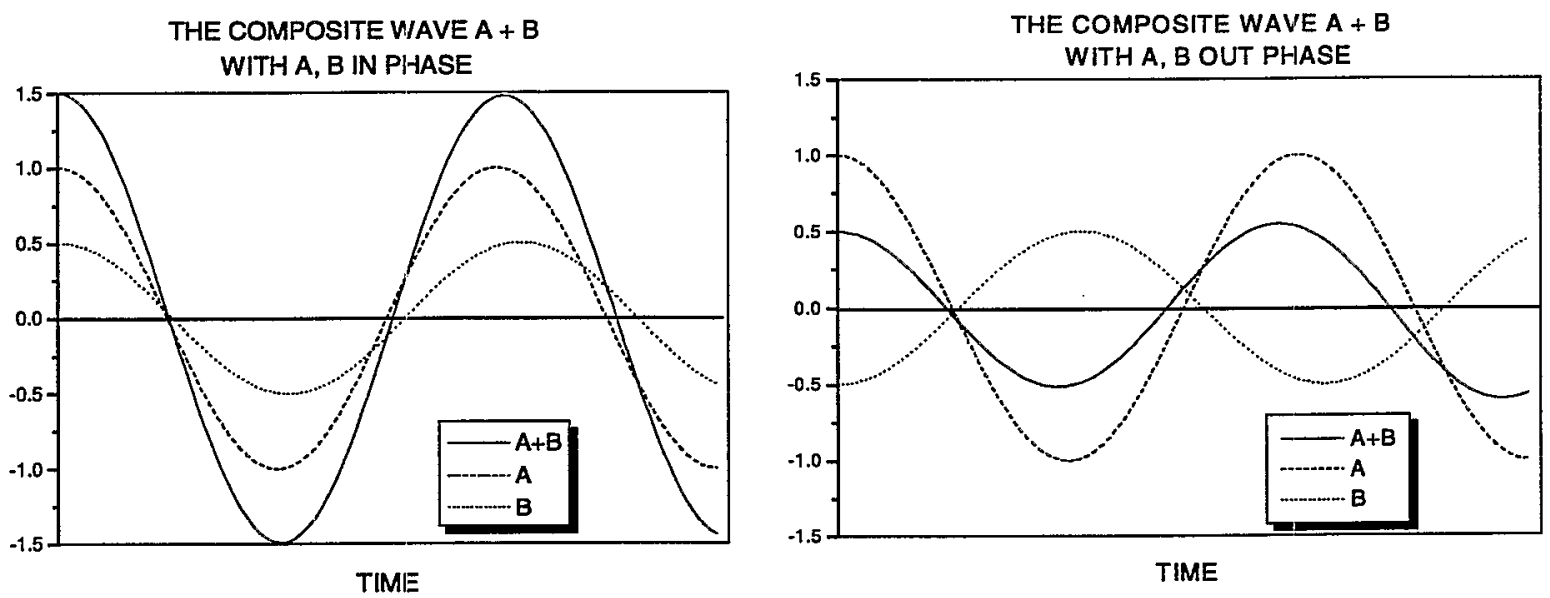

Fig.A1 The two waves and their composite wave

When the two waves are in phase, the period of the composite wave is in between the periods of its two components. But when they are out of phase, the period of composite wave is shorter than that of both components.

From (A22) and (A23) the frequency of the composite wave is: 


$$
\omega=\frac{\omega_{1}+\omega_{2}}{2}+\frac{\omega_{1}-\omega_{2}}{2} \cdot \frac{A_{p}}{\cos ^{2} \theta+A_{p}{ }^{2} \sin ^{2} \theta}
$$

Assuming $\omega_{2} / \omega_{1}=0.98$ and $A p=0.5$, Fig.A2 shows the relative frequency and the envelope of the composite wave, calculated by (A28) and (A3) respectively.
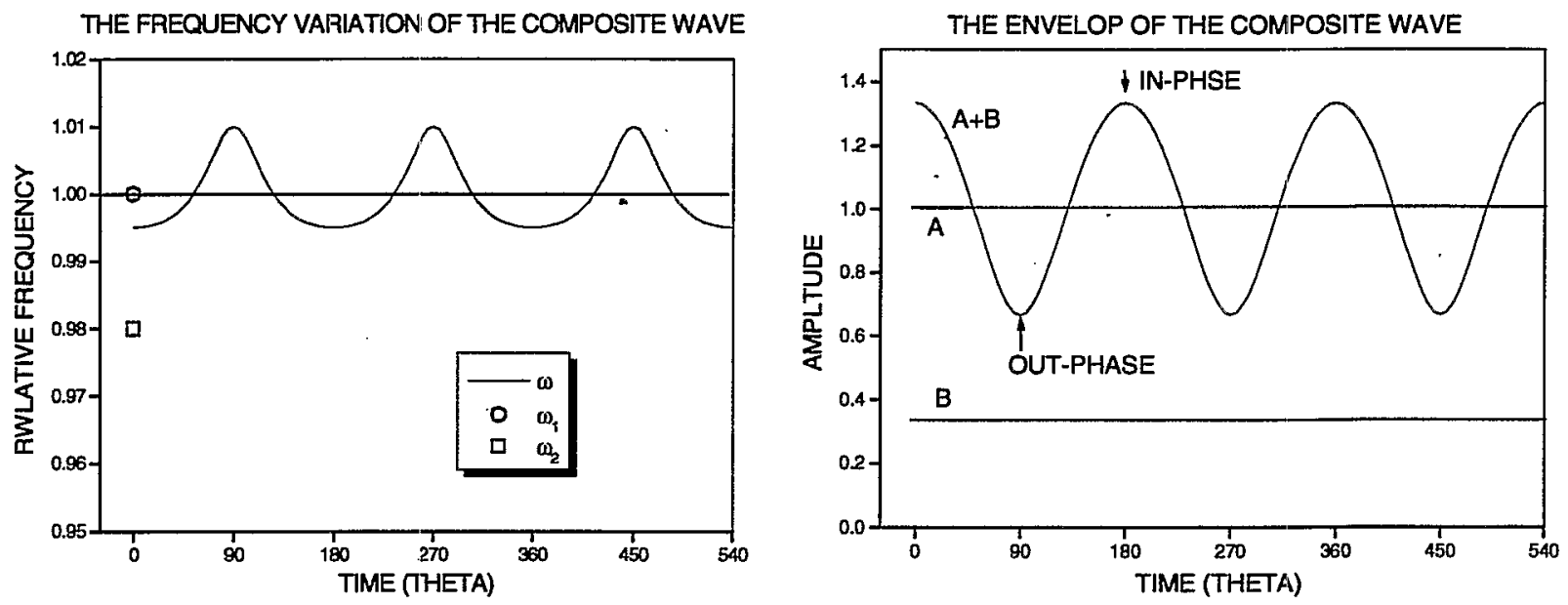

Fig.A2 The frequency and the envelop of the composite

It shows that when two waves are in-phase, the composite frequency is in between, the amplitude is the sum. When out of phase, the composite frequency is higher than that of both components, but the amplitude is their difference.

Fig.A3 shows the frequency variation with different $A_{p}$, a measure of amplitude ratio of two components (A12). If $A_{p}$ approaches zero, i.e. $A \approx B$, there is a peak at out of phase. In a limit case $B \rightarrow A$, $A_{p} \rightarrow 0$, the frequency deviation, the second tern in (A22), is zero except outphase points $\left(\theta=90^{\circ} \ldots\right)$, that is an irregularity, but meanwhile the amplitude is zero, so it is meaningless in practice.

In summary, we can conclude that:

The frequency of a beat wave formed by two components is swinging around the frequency of the stronger one. When two components are in phase, the

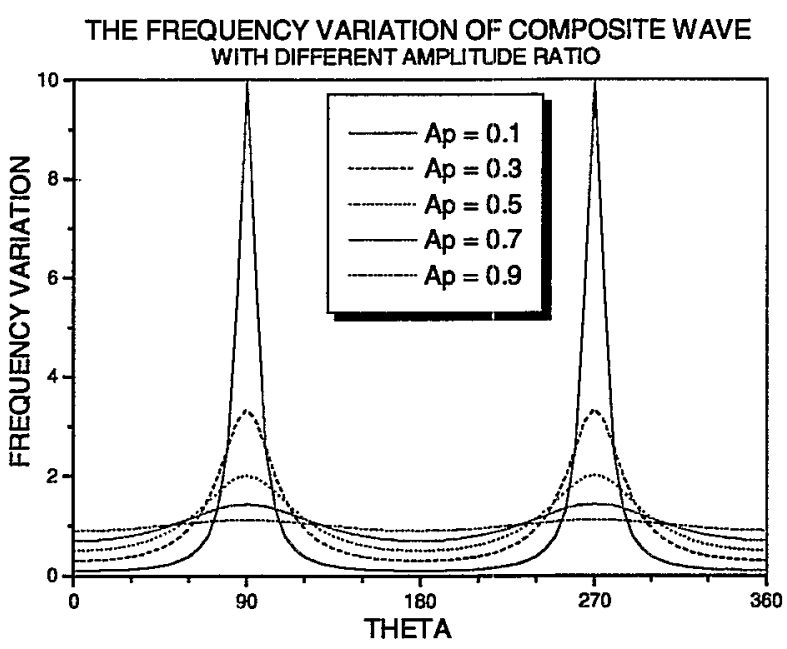

Fig.A3 The frequency variation composite frequency is toward that of the weaker component. When they are out of phase, the composite frequency is away from that of the weaker component. 